\title{
Development of personalised functional foods needs metabolic profiling
}

Article

Accepted Version

Claus, S. P. (2014) Development of personalised functional foods needs metabolic profiling. Current Opinion in Clinical Nutrition and Metabolic Care, 17 (6). pp. 567-573. ISSN 13631950 doi: https://doi.org/10.1097/MCO.0000000000000107 Available at https://centaur.reading.ac.uk/37544/

It is advisable to refer to the publisher's version if you intend to cite from the work. See Guidance on citing.

To link to this article DOI: http://dx.doi.org/10.1097/MCO.0000000000000107

Publisher: Lippincott, Williams \& Wilkins

All outputs in CentAUR are protected by Intellectual Property Rights law, including copyright law. Copyright and IPR is retained by the creators or other copyright holders. Terms and conditions for use of this material are defined in the End User Agreement.

\section{www.reading.ac.uk/centaur}

\section{CentAUR}

Central Archive at the University of Reading

Reading's research outputs online 
Development of personalised functional foods needs metabolic profiling

Sandrine Paule Claus

Department of Food \& Nutritional Sciences, The University of Reading, Whiteknights campus, PO Box 226, RG8 8JE

Email: s.p.claus@reading.ac.uk

Tel: $+44(0) 1183788717$ 


\begin{abstract}
Purpose of review

There is growing interest in applying metabolic profiling technologies to food science as this approach is now embedded into the foodomics toolbox. This review aims at exploring how metabolic profiling can be applied to the development of functional foods.
\end{abstract}

\title{
Recent findings
}

One of the biggest challenges of modern nutrition is to propose a healthy diet to populations worldwide that must suit high inter-individual variability driven by complex gene-nutrient-environment interactions. Although a number of functional foods are now proposed in support of a healthy diet, a one-size-fits-all approach to nutrition is inappropriate and new personalised functional foods are necessary. Metabolic profiling technologies can assist at various levels of the development of functional foods, from screening for food composition to identification of new biomarkers of food intake to support diet intervention and epidemiological studies.

\section{Summary}

Modern 'omics' technologies, including metabolic profiling, will support the development of new personalised functional foods of high relevance to twenty-firstcentury medical challenges such as controlling the worldwide spread of metabolic disorders and ensuring healthy ageing.

Keywords: functional foods, nutrition, metabolic profiling, metabolomics 


\section{Introduction}

The term "functional food" can be defined as a food product that confers some beneficial effects on health beyond the basic nutritional values of food [1]. As such, it is sometimes difficult to determine whether a food product should be classified or not as "functional". Functional food can therefore be either naturally rich or artificially enriched in substances (living organisms or nutrients) that are known for their beneficial effects such as probiotics, prebiotics, essential fatty acids, flavonoids, vitamins etc. In this regard, many natural products can be considered "functional" such as milk, numerous fruits and vegetables and oily fishes. To determine whether a food product has any specific health beneficial effect, one must measure the health impact of a complex food matrix following digestion (Figure 1). This can be achieved by measuring a number of physiological and biochemical parameters following diet intervention studies or epidemiologic surveys in relation with health outcome (e.g. HDL-LDL ratio in relation with cardiovascular disease). However, testing individuals with various genetic backgrounds results in high inter-individual variability and makes it difficult to detect subtle metabolic variations induced by a specific food intake. In addition, the gut microbiota is a highly variable environmental factor that must be considered as it inevitably interacts with the digestive process and may influence the overall outcome of ingested food.

These complex gene-nutrient-environment interactions are the same challenges that Linus Pauling faced in 1971 when he developed a new technique to enable "the thorough quantitative analysis of body fluids" that "might permit differential diagnosis of many diseases in a more effective way than is possible at the present time" [2]. In this pioneering publication, Pauling laid the foundations of a new analytical field that would lead to the development of modern metabolic profiling technologies [3]. Indeed, using a hypothesis-generating approach based on the untargeted measurement of the ensemble of metabolites present in a biological sample, also called metabolomics or metabolic fingerprinting, has proved extremely 
useful over the last decade in identifying potential disease markers [4] and is now embedded into the "foodomics" toolbox [5]. Hence, beyond its applications in the medical field, metabolic profiling can be applied at various stages of the development of new functional foods, as illustrated in Figure 1. This review will therefore explore how metabolic profiling has been recently applied to reveal the metabolic composition of potential functional food products or to support identification of new candidates. We will describe how it contributes to the assessment of the gut microbial metabolic activity and supports measurements of the metabolic outcomes of diet intervention studies and epidemiological surveys.

\section{Understanding food composition for screening of new functional ingredients}

Metabolomics is a powerful approach to metabolically characterise existing or potential functional food candidates. Not only can such approach contribute to improve our understanding of food product composition and bioaccessibility of their components, [6] but it may also reveal a number of unknown metabolites, particularly secondary metabolites in plants, that may be biologically active [7].

For instance, there is a growing interest in milk characterisation using modern metabolic profiling technologies (i.e. proton Nuclear Magnetic Resonance spectroscopy and Mass Spectrometry-based profiling) to better understand its formation and composition [8-11]. Milk is a complex food matrix that has been extensively studied for its major components (i.e. proteins, fat, lactose) and some minor components (i.e. minerals and vitamins). Only recently has the milk water soluble fraction drawn interest as it contains small molecular weight metabolites that are of nutritional importance, including numerous oligosaccharides [12,13]. Milk oligosaccharides are of particular importance since they are biologically active and can favour the growth of beneficial lactic acid bacteria in the gut. [reviewed here: [14]] Because oligosaccharides are found in relatively low concentration in nonhuman milk, most of the research effort over the last decade has been focused on the role of non-milk oligosaccharides such as plant-derived fructooligosaccharides 
(FOS) and enzymatically synthesized galactooligosaccharides (GOS). However, recent technological improvements using tandem mass spectrometry have facilitated characterisation of non-human milk oligosaccharides and promising results have been obtained using bovine milk oligosaccharides as new functional ingredients [1517].

There is also an emerging interest in assessing the fermentation products of fermented milk and dairy derivatives, as it has been recently demonstrated that these products stimulate the infant's immune system, even in absence of live bacteria $[18,19]$. Milk fermentation results in increased concentration of organic acids such as lactic acid, which are known to be beneficial as they lower the intestinal $\mathrm{pH}$ and protect against potential infections when fermentation occurs within the gastrointestinal tract. However, the active component of fermented milk derivatives is currently poorly understood. It is proposed that residual oligosaccharides produced from incomplete carbohydrate fermentation (i.e. sugars that are released by living organisms during the fermentation process but do not directly impart to the production of organic acids and other fermentation products) may have a prebiotic acitivity and contribute to the health promoting effect of these products. Another hypothesis is that bacteria themselves produce active compounds that would stimulate the immune system. These molecules are therefore of high importance as they may convene some health promoting activity and need to be further investigated. These are not specific to milk fermented derivatives and may be true for many other fermented food products, such as fermented soy derivatives. Metabolic profiling of fermented foods has been recognised as an appropriate strategy to identify potentially bioactive molecules [20]. Similarly, it has been recently attempted to use the same approach to rapidly screen for new functional food based on their prebiotic potential [21].

Finally, there has been a huge amount of research effort focused over the last decades on the identification of new probiotic species based on their effect on the 
immune system and their metabolism (e.g. ability to produce high amounts of butyric acid). However, this is a long and tedious work that can be expensive. An alternative approach is to use metabolomics to screen large numbers of potential probiotic species to classify them based on their metabolic activity [22]. In the future, such approach will certainly support the discovery of new bacteria and nutrients that promote human health.

\section{Assisting diet-intervention studies for better control of compliance}

The most challenging aspect in the development of functional food is to identify the bioactivity of a specific foodstuff. This is classically achieved using diet intervention studies where a number of volunteers are given a proposed functional food to consume for various amounts of time. In such experiments, it is of utmost importance to accurately record participants' diet over the course of the trial, which is classically achieved using a combination of three techniques: diet records, $24 \mathrm{~h}$ dietary recalls and food frequency questionnaires [23]. The main issue associated with these methods is that they are all based on self-reported information, which is subject to bias and misreporting [24]. There is therefore a need for a more accurate estimation of dietary intake, which can be achieved by identifying specific biomarkers of food intake that can be employed to correct self-reported dietary information and control for compliance in diet restricted studies.

In this regard, metabolic profiling has been widely applied in recent years to identify new biomarkers of food intake. Table 1 summarises the most recently published food biomarkers detected using a metabolomics approach. Some of these metabolites are now recognised markers, such as proline-betaine for citrus fruit consumption $[25,26]$, and these studies either confirm previously identified food consumption biomarkers or suggest new ones that will need further validation. The main weakness associated with the food biomarker approach is that many of these are not food specific since they can be detected in a large number of related food 
products. For example, hippuric acid and its derivatives are usually associated with vegetable intake as they derive from the degradation of plant polyphenols by the gut microbiota [27]. As a consequence, it is more accurate to refer to these substances as biomarkers of a food group, rather than a specific food product. Another approach is to use a combination (i.e. a profile) of markers that is more specific of a foodstuff or a diet. The research community is now increasingly aware of such limitations as illustrated by the recent publication of the Atherosclerosis Risk in Communities (ARIC) population study outcome [28]. Although researchers involved in the ARIC study reported some new food intake biomarkers, they clearly identified a set of markers associated with intake of "sugar-rich foods and beverages" (SRFB), which is also more meaningful in terms of biological interpretation. Similarly, a recent study used a metabolic profiling approach to identify various dietary traits in free-living subjects [29]. Although this is the first of its kind and further similar studies will be necessary to validate the profile of markers associated with each trait, this is a very encouraging work to better control for compliance in future diet intervention studies.

\section{Understanding food-mammalian metabolic interactions}

Some of the food intake biomarkers displayed in Table 1 are also endogenous compounds, such as scyllo-inositol, an ubiquitous component of cell

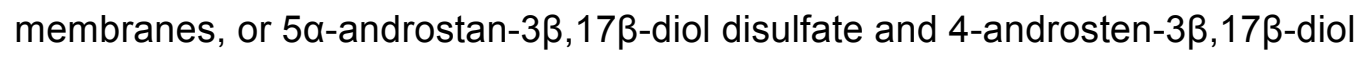
disulfate, which are sex hormone derivatives. The association of these chemicals with food intake may be due to confounding factors, but the ARIC study validated all potential biomarkers using an independent validation data set. Therefore, we may conclude that these chemicals are associated with the endogenous metabolic modulations triggered by the regular consumption of the food to which they were correlated. Indeed, nutrimetabonomics, defined as the quantitative measurement of the metabolic response of a living organism to a nutritional variation, is a powerful approach to generate new hypotheses of biological mechanisms underpinning food - 
health interactions. This metabolic profiling approach, which focuses on the modulations of endogenous metabolic pathways associated with diet interventions, has been successfully applied to improve our understanding of a number of natural products, nutrients and food groups [30-34]. For example, of the 23 metabolites associated with the SRFB food group in the ARIC study, five were found to be $\mathrm{Y}^{-}$ glutamyl dipeptides and five were related to the 2-hydroxybutyrate pathway [28]. The authors therefore concluded that this was indicative of oxidative stress associated with the consumption of SRFB foods.

Hence, the nutrimetabonomics approach is extremely useful to screen for markers of modulations of endogenous metabolic pathways in response to food intake. This is a powerful way to explore the impact of specific foods or nutrients on the systemic metabolism and has been widely applied to the investigation of the relationship between food/diet, gut microbiota and health. Indeed, the gut microbiota is an emerging environmental component at the crossroads of all environmental factors (i.e. under the influence of diet, lifestyle and exposome), which provides a reservoir of hundreds of thousands of unique extra genes [35-37]. It is therefore believed that manipulating the gut microbial ecosystem may result in health improvement of its host. A number of studies have used metabolic profiling technologies to assess the metabolic impact of the gut microbiota on its host, which have been reviewed here: $[38,39]$.

The gut microbiota is an extremely complex ecosystem and we are currently limited in our ability to measure the metabolic outcome of variations of specific groups or species of bacteria. To overcome this issue, a nutrimetabonomics approach has been recently applied to a prebiotic intervention study in obese women to link the bacterial content of human volunteers to metabolic markers of energy metabolism in plasma [40]. This illustrates that it is possible to decipher the impact of selected bacterial groups or species and their metabolism on the human host using such metabolic profiling approach [41]. Similarly, this can be applied to animal 
models where the metabolism of particular organs is of easier access. For example, a recent work profiled the hepatic metabolism and gut bacterial community in response to resistant starch intake in mice fed a high fat diet [42]. This revealed strong associations between hepatic amino acid metabolism and gut microbial modulation of high relevance for human nutrition.

Epidemiological surveys are also extremely useful to identify meaningful associations between diet and health. However, they often fail to detect subtle associations between nutrients and health due to the inevitable high degree of interindividual variation and poor diet records. Nonetheless, nutrimetabonomics has proved very useful in identifying metabolic markers of a healthy lifestyle. The International Collaborative Study on Macro-/Micronutrients and Blood Pressure (INTERMAP) study recently reported 12 urinary metabolites differentially observed in afro-americans versus non-hispanic white americans [43]. Of those, urinary hippuric acid was associated with lower blood pressure and higher polyphenol intake from fruits and vegetables. Hence, this NMR-based nutrimetabonomic study generated new hypotheses about possible links between urinary metabolites, food intake and cardiovascular health.

With the recent development of Genome Wide Association Studies with Metabolic traits (mGWAS), there is now the potential for identifying gene variants associated with specific metabolic functions. Although a few studies have attempted such approach to link the genome with the metabolome in a nutritional context, it is realistic to envisage that this strategy will lead to the identification of new genetic variants that predispose some individuals to nutrient deficiencies or to particular metabolic needs. Neverheless, beyond mGWAS, epigenetic and gut microbial variations must also be taken into account to develop a genuine personalised nutrition, which should be accompanied by personalised functional foods. Indeed, functional foods cannot be seen as a one-size-fits-all. This is already recognised by the research community working on modulation of the gut microbiota and huge 
efforts are currently pursued to stratify individuals according to their "enterotype" [44] to ultimately propose personalised probiotics. The same might be applied to other functional foods where inter-individual variability in metabolic capacities may affect the targetted benefit. Similarly, mGWAS have also been recently applied to plant science to unravel candidate genes associated with metabolic traits $[45,46]$. Such approach should be encouraged in the future in order to facilitate the production of functional crops carrying genetic variants associated with the production of beneficial compounds and/or nutrients. Assessing the metabolism is therefore essential to the success of such enterprise and metabolic profiling technologies will be indispensable to achieve this goal.

\section{Conclusion}

The twenty-first century is facing immense clinical challenges that include an overall ageing population along with a worldwide spread of metabolic disorders such as obesity and type 2 diabetes. The promotion of a healthy diet is an appropriate response to prevent such threats although high inter-individual variability driven by complex gene-nutrient-environment interactions calls for a more personalised approach that must be accompanied by the development of personalised functional foods. This can be supported by metabolic profiling as it can assist in developing new functional foods and nutrients at various stages of their elaboration. In the future, these technologies applied in conjonction with other 'omics' approaches to largescale mGWAS will play a major role to unravel the biological mechanisms underpinning inter-individual variability in response to food intake and identify new targets for functional ingredients.

\section{Key points:}

- Metabolic profiling is becoming increasingly popular to assess food composition, which facilitates the identification of new functional ingredients. 
- Metabolic profiling is a powerful approach to identify new biomarkers of food intake and assist diet intervention and epidemiological studies by offering a new way to control diet records and compliance.

- Genome Wide Association Studies with Metabolic traits are opening a new era in the unraveling of the complex gene-nutrient-environment interactions that will provide new targets for the development of functional foods.

\section{Acknowledgements}

The author would like to acknowledge funding from the European Union (FP7 grant number 613979).

\section{References}

1. Koletzko B, Aggett PJ, Bindels JG: Growth, development and differentiation: a functional food science approach. Br J Nutr 1998, 80:S5-S45.

2. Pauling L, Robinson AB, Teranishi R, Cary P: Quantitative analysis of urine vapor and breath by gas-liquid partition chromatography. Proc. Natl. Acad. Sci. U.S.A. 1971, 68:2374-2376.

3. Johnson $\mathrm{CH}$, Gonzalez FJ: Challenges and opportunities of metabolomics. J Cell Physiol 2012, 227:2975-2981.

4. Schlotterbeck G, Ross A, Dieterle F, Senn H: Metabolic profiling technologies for biomarker discovery in biomedicine and drug development. Pharmacogenomics 2006, 7:1055-1075.

5. Cifuentes A: Food analysis and Foodomics. J Chromatogr A 2009, 1216:7109-7109.

6. Pan X, Smith F, Cliff MT, Capozzi F, Mills ENC: The Application of Nutrimetabolomics to Investigating the Bioaccessibility of Nutrients in Ham Using a Batch in Vitro Digestion Model. Food and Nutrition Sciences 2014, 05:17-26.

7. * Abu Reidah IM, Contreras M, Arráez Román D, Fernández Gutiérrez A, Segura Carretero A: UHPLC-ESI-QTOF-MS-based metabolic profiling of Vicia faba L. (Fabaceae) seeds as a key strategy for characterization in foodomics. Electrophoresis 2014, 35:1571-1581.

This work used an untargeted metabolomic approach to characterise the 
metabolome of Vicia Faba seeds and revealed a number of new metabolites that had never been observed in this vegetable.

8. Maher AD, Hayes B, Cocks B, Marett L, Wales WJ, Rochfort SJ: Latent biochemical relationships in the blood-milk metabolic axis of dairy cows revealed by statistical integration of $1 \mathrm{H}$ NMR spectroscopic data. J Proteome Res 2013, 12:1428-1435.

9. Sundekilde U, Larsen L, Bertram H: NMR-Based Milk Metabolomics. Metabolites 2013, 3:204-222.

10. Melzer N, Wittenburg D, Hartwig S, Jakubowski S, Kesting U, Willmitzer $\mathrm{L}$, Lisec J, Reinsch N, Repsilber D: Investigating associations between milk metabolite profiles and milk traits of Holstein cows. Journal of Dairy Science 2013, 96:1521-1534.

11. Wittenburg D, Melzer N, Willmitzer L, Lisec J, Kesting U, Reinsch N, Repsilber D: Milk metabolites and their genetic variability. Journal of Dairy Science 2013, 96:2557-2569.

12. Smilowitz JT, O'Sullivan A, Barile D, German JB, Lonnerdal B, Slupsky CM: The Human Milk Metabolome Reveals Diverse Oligosaccharide Profiles. The Journal of Nutrition 2013, 143:1709-1718.

13. Praticò G, Capuani G, Tomassini A: Exploring human breast milk composition by NMR-based metabolomics. Natural Product Research 2014, 28:95-101.

14. Barile D, Rastall RA: Human milk and related oligosaccharides as prebiotics. Current opinion in biotechnology 2013, 24:214-219.

15. Aldredge DL, Geronimo MR, Hua S, Nwosu CC, Lebrilla CB, Barile D: Annotation and structural elucidation of bovine milk oligosaccharides and determination of novel fucosylated structures. Glycobiology 2013, 23:664-676.

16. Urashima T, Taufik E, Fukuda K: Recent advances in studies on milk oligosaccharides of cows and other domestic farm animals. Biosci Biotechnol Biochem 2013, 77:455-466.

17. Mehra R, Barile D, Marotta M, Lebrilla CB, Chu C, German JB: Novel High-Molecular Weight Fucosylated Milk Oligosaccharides Identified in Dairy Streams. PLoS ONE 2014, 9:e96040.

18. Campeotto F, Suau A, Kapel N, Magne F, Viallon V, Ferraris L, Waligora-Dupriet A-J, Soulaines $P$, Leroux B, Kalach $N$, et al.: A fermented formula in pre-term infants: clinical tolerance, gut microbiota, down-regulation of faecal calprotectin and upregulation of faecal secretory IgA. Br J Nutr 2011, 105:1843-1851.

19. Morisset M, Aubert-Jacquin C, Soulaines P, Moneret-Vautrin D-A, Dupont C: A non-hydrolyzed, fermented milk formula reduces 
digestive and respiratory events in infants at high risk of allergy. Eur J Clin Nutr 2011, 65:175-183.

20.* Mozzi F, Ortiz ME, Bleckwedel J, De Vuyst L, Pescuma M:

Metabolomics as a tool for the comprehensive understanding of fermented and functional foods with lactic acid bacteria. Food Research International 2013, 54:1152-1161.

This presents an extensive review of the use of metabolomics in the study of fermented food. It includes a discussion of its use in fermented soy foods, cheeses, wines, coca bean fermentation and probiotics.

21. Date $Y$, Nakanishi $Y$, Fukuda S, Nuijima Y, Kato T, Umehara M, Ohno $\mathrm{H}$, Kikuchi J: In vitro evaluation method for screening of candidate prebiotic foods. Food Chemistry 2014, 152:251-260.

22. Perez-Fons L, Bramley PM, Fraser PD: The optimisation and application of a metabolite profiling procedure for the metabolic phenotyping of Bacillus species. Metabolomics 2014, 10:77-90.

23. Webb D, Leahy MM, Milner JA, Allison DB, Dodd KW, Gaine PC, Matthews RAJ, Schneeman BO, Tucker KL, Young SS: Strategies to optimize the impact of nutritional surveys and epidemiological studies. Adv Nutr 2013, 4:545-547.

24. Johnson RK: Dietary intake--how do we measure what people are really eating? Obes. Res. 2002, 10 Suppl 1:63S-68S.

25. Heinzmann SS, Brown IJ, Chan Q, Bictash M, Dumas M-E, Kochhar S, Stamler J, Holmes E, Elliott P, Nicholson JK: Metabolic profiling strategy for discovery of nutritional biomarkers: proline betaine as a marker of citrus consumption. Am J Clin Nutr 2010, 92:436-443.

26. Lloyd AJ, Beckmann M, Favé G, Mathers JC, Draper J: Proline betaine and its biotransformation products in fasting urine samples are potential biomarkers of habitual citrus fruit consumption. Br J Nutr 2011, 106:812-824.

27. Lees HJ, Swann JR, Wilson ID, Nicholson JK, Holmes E: Hippurate: The Natural History of a Mammalian-Microbial Cometabolite. $J$ Proteome Res 2013, 12:1527-1546.

28. ** Zheng Y, Yu B, Alexander D, Steffen LM, Boerwinkle E: Human metabolome associates with dietary intake habits among african americans in the atherosclerosis risk in communities study. Am. J. Epidemiol. 2014, 179:kwu073v1-kwu073.

This interesting publication reports the outcome of the ARIC study where a number of food-related biomarkers were associated with metabolic disruptions. In particular, some markers of oxidative stress were associated with high intake of sugar-rich foods and beverages. 
29.** Andersen MBS, Rinnan Å, Manach C, Poulsen SK, Pujos-Guillot E, Larsen TM, Astrup A, Dragsted LO: Untargeted metabolomics as a screening tool for estimating compliance to a dietary pattern. $J$ Proteome Res 2014, 13:1405-1418.

This pioneering study is the first of its kind to use a metabolic profiling approach to estimate compliance to a complex diet and identify dietary patterns in free-living subjects following a New Nordic Diet or and Average Danish Diet.

30. Bondia-Pons I, Cañellas N, Abete I, Rodríguez MÁ, Perez-Cornago A, Navas-Carretero S, Zulet MÁ, Correig X, Martínez JA: NutriMetabolomics: Subtle Serum Metabolic Differences in Healthy Subjects by NMR-Based Metabolomics after a Short-Term Nutritional Intervention with Two Tomato Sauces. Mol Nutr Food Res. 2013, 17:611-618.

31. Andersen M-BS, Reinbach HC, Rinnan Å, Barri T, Mithril C, Dragsted LO: Discovery of exposure markers in urine for Brassicacontaining meals served with different protein sources by UPLCqTOF-MS untargeted metabolomics. Metabolomics 2013, 9:984-997.

32. Orozco-Solano Ml, Priego-Capote F, de Castro MDL: Analysis of esterified and nonesterified fatty acids in serum from obese individuals after intake of breakfasts prepared with oils heated at frying temperature. Anal Bioanal Chem 2013, 405:6117-6129.

33. Rudkowska I, Paradis A-M, Thifault E, Julien P, Barbier O, Couture P, Lemieux S, Vohl M-C: Differences in metabolomic and transcriptomic profiles between responders and non-responders to an $n-3$ polyunsaturated fatty acids (PUFAs) supplementation. Genes Nutr 2013, 8:411-423.

34. ** Johnson CH, Slanař O, Krausz KW, Kang DW, Patterson AD, Kim J-H, Luecke H, Gonzalez FJ, Idle JR: Novel metabolites and roles for $\alpha$ tocopherol in humans and mice discovered by mass spectrometrybased metabolomics. Am J Clin Nutr 2012, 96:818-830.

This original study employed a nutrimetabonomics approach to reveal new intermediary metabolites in the endogenous metabolism of alphatocopherol. The authors also compared administration of synthetic alpha-tocopherol supplement to administration of a similar dose as a whole food (almonds).

35. Nicholson JK, Holmes E, Wilson ID: Opinion: Gut microorganisms, mammalian metabolism and personalized health care. Nature Reviews Microbiology 2005, 3:431-438.

36. Maurice CF, Haiser HJ, Turnbaugh PJ: Xenobiotics Shape the Physiology and Gene Expression of the Active Human Gut Microbiome. Cell 2013, 152:39-50. 
37. Ursell LK, Haiser HJ, Van Treuren W, Garg N, Reddivari L, Vanamala J, Dorrestein PC, Turnbaugh PJ, Knight R: The Intestinal Metabolome: An Intersection Between Microbiota and Host. Gastroenterology 2014, 146:1470-1476.

38. Claus SP, Swann JR: Nutrimetabonomics: Applications for Nutritional Sciences, with Specific Reference to Gut Microbial Interactions. Annu Rev Food Sci Technol 2013, 4:381-399.

39. Swann JR, Claus SP: Nutrimetabonomics: nutritional applications of metabolic profiling. Science Progress 2014, 97:41-47.

40. ** Dewulf EM, Cani PD, Claus SP, Fuentes S, Puylaert PGB, Neyrinck AM, Bindels LB, de Vos WM, Gibson GR, Thissen J-P, et al.: Insight into the prebiotic concept: lessons from an exploratory, double blind intervention study with inulin-type fructans in obese women. Gut 2013, 62:1112-1121.

This is the first study to link gut microbiota to specific circulating metabolites involved in the energy pathway in humans. This was achieved using a metabolic profiling approach in a prebiotic intervention trial in obese women.

41. Tilg H, Gasbarrini A: Prebiotics for obesity: a small light on the horizon? Gut 2013, 62:1096-1097.

42. Kieffer D, Martin R, Marco M, Kim E, Keenan M: Resistant starch significantly alters gut microbiota and liver metabolome in mice fed a high fat diet. The FASEB Journal 2014, 28:822.13.

43. * Stamler J, Brown IJ, Yap IKS, Chan Q, Wijeyesekera A, Garcia-Perez I, Chadeau-Hyam M, Ebbels TMD, De lorio M, Posma J, et al.: Dietary and urinary metabonomic factors possibly accounting for higher blood pressure of black compared with white Americans: results of International Collaborative Study on macro-/micronutrients and blood pressure. Hypertension 2013, 62:1074-1080.

This multicentre population-based study identified biomarkers of high-blood pressure that were associated with specific dietary patterns.

44. ${ }^{* *}$ Arumugam M, Raes J, Pelletier E, Le Paslier D, Yamada T, Mende DR, Fernandes GR, Tap J, Bruls T, Batto J-M, et al.: Enterotypes of the human gut microbiome. Nature 2011, 473:174-180.

This important study used a metagenomics approach to identify 3 enterotypes among human populations, independently of their geographic location. This is a fundamental work that demonstrates that individuals can be stratified based on the metabolic potential of their microbial community.

45. Wen W, Li D, Li X, Gao Y, Li W, Li H, Liu J, Liu H, Chen W, Luo J, et al.: Metabolome-based genome-wide association study of maize kernel leads to novel biochemical insights. Nat Comms 2014, 


\section{5:3438.}

46. Chen W, Gao Y, Xie W, Gong L, Lu K, Wang W, Li Y, Liu X, Zhang H, Dong $\mathrm{H}$, et al.: Genome-wide association analyses provide genetic and biochemical insights into natural variation in rice metabolism. Nat. Genet. 2014, 46:714-721.

47. ** Scalbert A, Brennan L, Manach C, Andres-Lacueva C, Dragsted LO, Draper J, Rappaport SM, van der Hooft JJ, Wishart DS: The food metabolome: a window over dietary exposure. Am J Clin Nutr 2014, 99:1286-1308.

This is an extensive review that covers in depth the use of metabolic profiling to characterise food composition and identify biomarkers of food intake.

48. Llorach R, Medina S, García Viguera C, Zafrilla P, Abellán J, Jauregui O, Tomás Barberán FA, Gil Izquierdo A, Andres-Lacueva C: Discovery of human urinary biomarkers of aronia-citrus juice intake by HPLC-q-TOF-based metabolomic approach. Electrophoresis 2014, 35:1599-1606.

49. Stanstrup J, Rasmussen JE, Ritz C, Holmer-Jensen J, Hermansen K, Dragsted LO: Intakes of whey protein hydrolysate and whole whey proteins are discriminated by LC-MS metabolomics. Metabolomics 2013, doi:10.1007/s11306-013-0607-9.

50. Johansson-Persson A, Barri T, Ulmius M, Önning G, Dragsted LO: LCQTOF/MS metabolomic profiles in human plasma after a 5-week high dietary fiber intake. Anal Bioanal Chem 2013, 405:4799-4809.

51. Bondia-Pons I, Barri T, Hanhineva K, Juntunen K, Dragsted LO, Mykkänen H, Poutanen K: UPLC-QTOF/MS metabolic profiling unveils urinary changes in humans after a whole grain rye versus refined wheat bread intervention. Mol. Nutr. Food Res. 2013, 57:412422.

52. Garcia-Aloy M, Llorach R, Urpi-Sarda M, Tulipani S, Estruch R, Martínez-González MA, Corella D, Fitó M, Ros E, Salas-Salvadó J, et al.: Novel multi-metabolite prediction of walnut consumption by a urinary biomarker model in a free-living population. J Proteome Res 2014, doi:10.1021/pr500425r. 


\section{Figure legend}

Figure 1: Metabolic profiling can be applied at various stages of the development of new functional foods. The metabolic composition of complex food matrices can be characterised using metabolic profiling technologies. Untargeted approaches can facilitate identification of new metabolites of particular interest and contribute to the development of new functional ingredients. Gut microbiota is an inevitable partner whith a degree of inter-individual variability that must be considered in the elaboration of functional foods, as well as being a potential target. Gut microbial metabolic activity is rapidly screened using metabolic profiling technologies. Diet intervention studies and epidemiological surveys require accurate records of food intake and control for diet compliance, which can be achieved by relying on the measurement of metabolic biomarkers of food intake. The metabolic outcome of diet intervention studies and Genome-Wide Association Studies with Metabolic traits (mGWAS) are assisted by metabolic profiling. 
Table 1: Potential biomarkers of food intake.

\begin{tabular}{|c|c|c|c|c|}
\hline Food Product & Biomarker & Analytical Method & \begin{tabular}{|l|} 
Biological \\
Matrix \\
\end{tabular} & Ref \\
\hline Aronia-Citrus juice & Proline-betaine; ferulic acid; mercapturate derivatives & HPLC-qTOF-MS & Urine & [48] \\
\hline Brassica oleraceae & $\mathrm{N}$-acetyl isothiocyanates; cysteine isothiocyanates & HPLC-qTOF-MS & Urine & {$[31]$} \\
\hline $\begin{array}{l}\text { Caseinoglycomacropeptide } \\
\text { (from milk) }\end{array}$ & $\begin{array}{l}\text { a-ketoisovalerate, } \alpha \text {-hydroxybutyrate and isomers; } \alpha \text {-hydroxyisovalerate; } \\
\text { a-keto-3-methylvalerate }\end{array}$ & LC-qTOF-MS & Plasma & [49] \\
\hline Citrus fruits & Proline-betaine; hesperetin-glucuronide & UPLC-qTOF-MS & Urine & [29] \\
\hline Cocoa & $\begin{array}{l}\text { Theobromine, 6-amino-5-[N-methylformylamino]-1-methyluracil; 7- } \\
\text { methyluric acid }\end{array}$ & UPLC-qTOF-MS & Urine & [29] \\
\hline Coffee & $\begin{array}{l}\text { Quinate; paraxanthine; 5-acetylamino-6-amino-3-methyluracil; 1,7- } \\
\text { dimethylurate; a-methylurate; 1-methylxanthine; caffeine; 1,3,7- } \\
\text { trimethylurate; 7-methylxanthine }\end{array}$ & GC-MS and LC-MS & Serum & [28] \\
\hline Egg & Docosapentaenoate & GC-MS and LC-MS & Serum & {$[28]$} \\
\hline Fiber intake & 2,6-dihydroxybenzoic acid; 2-aminophenol sulfate & LC-qTOF-MS & Plasma & [50] \\
\hline \multirow[t]{2}{*}{ Fish \& Seafood } & Eicosapentaenoate, docosahexaenoate & GC-MS and LC-MS & Serum & {$[28]$} \\
\hline & TMAO & UPLC-qTOF-MS & Urine & [31] \\
\hline Fruit juice & $\begin{array}{l}\text { Glycerate; stachydrine; N-methylproline; threonate; scyllo-inositol; } \\
\text { homostachydrine }\end{array}$ & GC-MS and LC-MS & Serum & [28] \\
\hline Nuts \& Peanut butter & 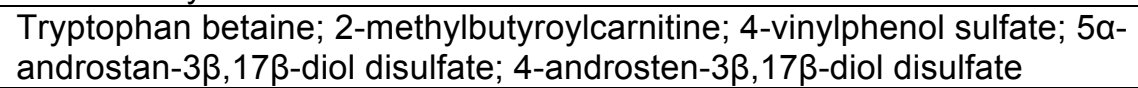 & GC-MS and LC-MS & Serum & {$[28]$} \\
\hline Red Cabbage & Hippuric acid derivatives; acetyl-cysteine conjugates of isothiocyanates; & UPLC-qTOF-MS & Urine & [29] \\
\hline Rye bread & $\begin{array}{l}\text { 2-aminophenol sulfate; nonanedioic acid; indolylacryloylglycine; } \\
\text { enterolactone glucuronide; ferulic acid-4-O-sulfate }\end{array}$ & UPLC-qTOF-MS & Urine & {$[51]$} \\
\hline \multirow[t]{2}{*}{ Walnut } & Indolic compounds; conjugated urolithin & HPLC-qTOF-MS & Urine & {$[52]$} \\
\hline & 5-hydroxyindole-3-acetic acid & UPLC-qTOF-MS & Urine & [29] \\
\hline Refined Wheat bread & Ascorbic acid (industrial additive); Quinic acid & UPLC-qTOF-MS & Urine & [51] \\
\hline Whey concentrate hydrolysate & $\begin{array}{l}\text { pGlu-Leu; Methionine sulfoxide; N-phenylacetyl-methionine; N- } \\
\text { phenylacetyl-methionine sulfoxide; pGlu-Val }\end{array}$ & LC-qTOF-MS & Plasma & {$[49]$} \\
\hline
\end{tabular}

Modified from [47]. 